International Journal of Pure and Applied Mathematics

Volume 98 No. 2 2015, 193-210

ISSN: 1311-8080 (printed version); ISSN: 1314-3395 (on-line version)

url: http://www.ijpam.eu

doi: http://dx.doi.org/10.12732/ijpam.v98i2.3

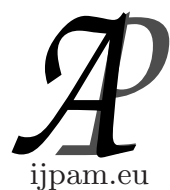

\title{
OBTAINING A COMPROMISE SOLUTION OF \\ A MULTI OBJECTIVE FIXED CHARGE PROBLEM IN A FUZZY ENVIRONMENT
}

\author{
M. Upmanyu ${ }^{1 \S}$, R.R. Saxena ${ }^{2}$ \\ ${ }^{1}$ Department of Mathematics \\ Faculty of Mathematical Sciences \\ University of Delhi \\ Delhi, 110007, INDIA \\ ${ }^{2}$ Department of Mathematics \\ Deen Dayal Upadhyaya College \\ Karampura, Delhi, 110015, INDIA
}

\begin{abstract}
The fixed charge problem is an important application of the class of mixed integer programming problems, wherein a charge is associated with performing an activity at a nonzero level which does not depend on the level of the activity. In real world problems, the decision maker is usually unable to provide precise information regarding these fixed costs and other coefficients in the objective function. This imprecision here is dealt with the help of fuzzy numbers. This paper presents a fuzzy programming approach to determine a compromise solution of a fixed charge problem with several fuzzy objective functions. The procedure for solving the fixed charge problem is based upon the Balinski approximation solution method for a fixed cost transportation problem. A numerical example is presented to illustrate the proposed method.
\end{abstract}

AMS Subject Classification: 90C08, 90C11, 90C29, 90C70

Key Words: fixed charge problem, multiobjective programming, fuzzy objective function, compromise solution

Received: August 18, 2014

(C) 2015 Academic Publications, Ltd.

${ }^{\S}$ Correspondence author

url: www.acadpubl.eu 


\section{Introduction}

The fixed charge problem (FCP) is an important application of the class of mixed integer programming problems, which form the basis of many business and industry problems wherein a charge is associated with performing an activity at a nonzero level which does not depend on the level of the activity. The existence of these fixed charges associated with the activities in the objective function produce a nonlinear programming problem. The fixed charge can also be expressed as the set up time of getting a machine into service or the fixed elapsed time before a certain operation can commence. In 1968, Hirsch and Dantzig [7] first introduced this problem and found a solution by examining the extreme points of the convex set of feasible solutions. The FCP has been widely applied in many decision-making and optimization problems like the warehouse or plant location decisions, wherein there is a charge associated with opening the facility; and in transportation problems where there are fixed charges for transporting goods between supply points and demand points.

Till date the methods obtained to solve the FCP are mainly approximate methods developed by Cooper [4], Murty [10], Cooper and Drebes [5], Walker [13] which are all based on adjacent extreme point algorithms. For small problems, Steinberg [11] provided an exact method based on the branch and bound method. The fixed cost transportation problem (FCTP) [2] is an important modern application of the FCP mainly solved by ranking of the extreme points and the branch and bound method. This problem reduces to that of minimising a concave function over a bounded convex set and the resulting optimum is taken at one or more of the extreme points of the feasible region. In [1], Adlakha and Kowalski use the Balinski approximation method introduced for the fixed charge transportation problem and apply the same for solving the fixed charge problem.

In real world situations, the parameters in the objectives are supplied according to the decision makers' requirements. In most cases he is unable to provide this information precisely, and to deal with this imprecision we formulate these parameters as fuzzy numbers, in particular as triangular fuzzy numbers. In other words the objective function is fuzzified and leverage is provided to the decision maker to operate.

Since in practical situations the set of all efficient solutions is not always necessary to obtain, we develop a procedure for finding a compromise solution. The fuzzy programming approach introduced by Zimmermann [14] as an application of fuzzy linear programming to the linear vector maximum problem, in which fuzzy set theory has been applied to multi criteria decision making 
problems using a linear membership function, obtains efficient solutions as well as a compromise solution [9]; one which not only satisfies the decision maker's preferences, but is also preferred to all other solutions. Methods for obtaining a compromise solution of multi objective transportation problems have been developed in recent times in which not only the fuzzy min operator, but various other fuzzy aggregation operators are used [8]. To the authors' knowledge, no work has yet been done in developing a solution procedure for solving a multi objective fixed charge problem with objective functions having fuzzy parameters. In this paper we intend to provide the required algorithm and a compromise solution for the same. Preliminary concepts of fuzzy numbers and the FCP are introduced in Section 2. The problem under discussion is formulated, and the Balinski approximation is extended for FCPs in Section 3. The solution procedure developed in Section 4 is supported by a numerical example in Section 5.

\section{Preliminaries}

In this section we review some fundamentals of fuzzy numbers which we will use in the paper.

Definition 1. Fuzzy Set A fuzzy set $\tilde{A}$ of the referential set $X$ is characterized by a membership function $\mu_{\tilde{A}}$ which is valued in [0,1], that is $\mu_{\tilde{A}}$ : $X \rightarrow[0,1]$ with $\mu_{\tilde{A}}(x)$ representing the degree of membership of $x \in X$ in $\tilde{A}$.

Here we consider $X=\mathbb{R}$, the real line. We shall use the most common definition of fuzzy numbers given by Dubois and Prade [6].

Definition 2. Fuzzy Number A fuzzy number $\tilde{A}$ is a convex normalized fuzzy set of the real line $\mathbb{R}$ such that:

(i) $\exists$ unique $x_{0} \in \mathbb{R}$ such that $\mu_{\tilde{A}}\left(x_{0}\right)=1$, and

(ii) $\mu_{\tilde{A}}$ is piecewise continuous.

In this paper triangular fuzzy numbers of the form $\tilde{A}=\left(a^{o}, a^{m}, a^{p}\right)$ having a triangular possibility distribution are considered, where $a^{m}$ is the most possible value that definitely belongs to the set of most available values (possibility $=1$, if normalised), and $a^{p}$ (the most pessimistic value) and $a^{o}$ (the most optimistic value) are the least possible values which have a very low likelihood of belonging to the set of available values.

Let us denote the set of fuzzy numbers by $F(\mathbb{R})$. The basic arithmetic operations between two triangular fuzzy numbers are as follows [14]. 
Let $\tilde{A}=(a, b, c), \tilde{B}=(x, y, z) \in F(\mathbb{R})$, then

(i) Addition: $(\tilde{A} \oplus \tilde{B})=(a+x, b+y, c+z)$

(ii) Scalar Multiplication: $(k \otimes \tilde{A})= \begin{cases}(k a, k b, k c) & \text {, if } k>0 \\ (k c, k b, k a) & \text {, if } k<0\end{cases}$

(iii) Multiplication: $(\tilde{A} \otimes \tilde{B})=(\min \{a x, a z, c x, c z\}, b y, \max \{a x, a z, c x, c z\})$

(iii) Division: $(\tilde{A} \oslash \tilde{B})=(\min \{a / x, a / z, c / x, c / z\}, b / y, \max \{a / x, a / z, c / x, c / z\})$

(v) Non negativity: $\tilde{A}$ is said to be a non negative triangular fuzzy number iff $a \geq 0$.

Due to the absence of any linear ordering in the set $F(\mathbb{R})$, two fuzzy numbers cannot be compared. To enable such a comparison, a simple concept of ranking functions is introduced. A ranking function is a mapping $R: F(\mathbb{R}) \rightarrow \mathbb{R}$ which maps each fuzzy number into a point on the real line where a natural order exists. This ordering is defined as

$$
\begin{aligned}
& \tilde{A} \succcurlyeq \tilde{B} \text { if and only if } R(\tilde{A}) \geq R(\tilde{B}), \\
& \tilde{A} \succ \tilde{B} \text { if and only if } R(\tilde{A})>R(\tilde{B}), \\
& \tilde{A} \approx \tilde{B} \text { if and only if } R(\tilde{A})=R(\tilde{B}),
\end{aligned}
$$

where $\tilde{A}, \tilde{B} \in F(\mathbb{R})$. Also we write $\tilde{A} \preccurlyeq \tilde{B}$ iff $\tilde{B} \succcurlyeq \tilde{A}$, and $\tilde{A} \prec \tilde{B}$ iff $\tilde{B} \succ \tilde{A}$.

Note For computational purposes, we have defined a linear ranking function for a triangular fuzzy number as

$$
R(\tilde{A})=1 / 3(a+b+c), \text { for } \tilde{A}=(a, b, c) \in F(\mathbb{R}) .
$$

\section{Theoretical Development}

\subsection{Problem Formulation}

Consider the mathematical model of the multi objective Fixed Charge Problem having imprecise objective functions (FMFP) below 
(FMFP) Minimize $\tilde{Z}=\left(\tilde{Z}_{1}, \tilde{Z}_{2}, \ldots, \tilde{Z}_{p}\right)$

$$
\begin{gathered}
\text { subject to } \quad \sum_{j=1}^{n} a_{i j} x_{j}=b_{i}, \quad i \in I=\{1,2, \ldots, m\} \\
x_{j} \geq 0, \quad j \in J=\{1,2, \ldots, n\} \\
\tilde{Z}_{k}=\sum_{j=1}^{n}\left(\tilde{c}_{k j} x_{j}+\tilde{f}_{k j} y_{j}\right), \quad k \in K=\{1,2, \ldots, p\}
\end{gathered}
$$

Here where $p$ is the number of objectives, $\tilde{c}_{k j}$ is the fuzzy cost and $\tilde{f}_{k j}$ is the fuzzy fixed charge associated with the variable $x_{j},(k \in K, j \in J)$ and

$$
y_{j}= \begin{cases}0 & \text { if }, x_{j}=0 \\ 1 & \text { if }, x_{j}>0\end{cases}
$$

Let us define the set of feasible solutions of FMFP as $S=\left\{X \in \mathbb{R}^{n}: \sum_{j=1}^{n} a_{i j} x_{j}=b_{i}, x_{j} \geq 0, i \in I, j \in J\right\}$. It is also assumed that the parameters $a_{i j}, b_{i}, \tilde{c}_{k j}$ and $\tilde{f}_{k j}$ are non negative for all $i \in I, j \in J, k \in K$.

Using ranking functions, we introduce the concept of Pareto optimal solutions or R - efficient solutions of the multi objective problem FMFP by extending the ordinary Pareto optimality definition.

Definition 3. $\mathbf{R}$ - efficient Solution A solution $X^{*} \in S$ is said to be an $R$ - efficient solution of FMFP if and only if there does not exist another solution $X \in S$ such that

$$
\begin{gathered}
\tilde{Z}_{k}(X) \preccurlyeq \tilde{Z}_{k}\left(X^{*}\right) \text { for all } k \in K \\
\text { and } \tilde{Z}_{r}(X) \prec \tilde{Z}_{r}\left(X^{*}\right) \text { for at least one } r \in\{1,2, \ldots, p\}
\end{gathered}
$$

In other words, $X^{*}$ is an $\mathrm{R}$ - efficient solution of (FMFP) if and only if there does not exist another $X \in S$ such that

$$
\begin{gathered}
R\left(\tilde{Z}_{k}(X)\right) \leq R\left(\tilde{Z}_{k}\left(X^{*}\right)\right) \text { for all } k \in K \\
\text { and } R\left(\tilde{Z}_{r}(X)\right)<R\left(\tilde{Z}_{r}\left(X^{*}\right)\right) \text { for at least one } r \in\{1,2, \ldots, p\},
\end{gathered}
$$

where $\mathrm{R}$ is a ranking function. The set of all Pareto optimal solutions $\mathrm{E}$ is called the complete solution of the problem. 
Now each $\tilde{c}_{k j}$ and $\tilde{f}_{k j}$ is a triangular fuzzy number defined as $\tilde{c}_{k j}=\left(c_{k j}^{o}, c_{k j}^{m}, c_{k j}^{p}\right)$, and $\tilde{f_{k j}}=\left(f_{k j}^{o}, f_{k j}^{m}, f_{k j}^{p}\right), j \in J$ and $k \in K$.

$$
\text { Then, } \begin{aligned}
\tilde{Z}_{k} & =\sum_{j=1}^{n}\left(\left(c_{k j}^{o}, c_{k j}^{m}, c_{k j}^{p}\right) x_{j}+\left(f_{k j}^{o}, f_{k j}^{m}, f_{k j}^{p}\right) y_{j}\right) \\
& =\sum_{j=1}^{n}\left(\left(c_{k j}^{o} x_{j}+f_{k j}^{o} y_{j}\right),\left(c_{k j}^{m} x_{j}+f_{k j}^{m} y_{j}\right),\left(c_{k j}^{p} x_{j}+f_{k j}^{p} y_{j}\right)\right) \\
& =\left(\sum_{j=1}^{n}\left(c_{k j}^{o} x_{j}+f_{k j}^{o} y_{j}\right), \sum_{j=1}^{n}\left(c_{k j}^{m} x_{j}+f_{k j}^{m} y_{j}\right), \sum_{j=1}^{n}\left(c_{k j}^{p} x_{j}+f_{k j}^{p} y_{j}\right)\right) \\
& =\left(Z_{k}^{o}, Z_{k}^{m}, Z_{k}^{p}\right), \quad \text { say }
\end{aligned}
$$

Clearly $\tilde{Z}_{k}$ is also a triangular fuzzy number, and so each fuzzy objective function is in turn a triangular fuzzy number which is to be minimised. Since the ranking function of $\tilde{Z}_{k}$ is taken to be $R\left(\tilde{Z}_{k}\right)=1 / 3\left(Z_{k}^{o}+Z_{k}^{m}+Z_{k}^{p}\right)$, thus in order to minimise $\tilde{Z}_{k}$ we can equivalently minimise each of its three components simultaneously. It is easy to see that the smaller the values of the three components, the smaller will be the value of $R\left(\tilde{Z}_{k}\right)$ and the more preferred the solution will be.

Hence based on the above, we define the minimum of the fuzzy valued objective function $\tilde{Z}_{k}$ as

$$
\operatorname{Min} \tilde{Z}_{k}=\operatorname{Min}\left(Z_{k}^{o}, Z_{k}^{m}, Z_{k}^{p}\right), \quad k \in K
$$

Then the given problem FMFP can be reformulated to the equivalent multicriteria fixed charge problem MFCP below with three objectives corresponding to each $k \in K$

$$
\begin{aligned}
& \operatorname{Min} Z_{k 1}=\operatorname{Min} Z_{k}^{o}=\operatorname{Min} \sum_{j=1}^{n}\left(c_{k j}^{o} x_{j}+f_{k j}^{o} y_{j}\right) \\
& \operatorname{Min} Z_{k 2}=\operatorname{Min} Z_{k}^{m}=\operatorname{Min} \sum_{j=1}^{n}\left(c_{k j}^{m} x_{j}+f_{k j}^{m} y_{j}\right) \\
& \operatorname{Min} Z_{k 3}=\operatorname{Min} Z_{k}^{p}=\operatorname{Min} \sum_{j=1}^{n}\left(c_{k j}^{p} x_{j}+f_{k j}^{p} y_{j}\right)
\end{aligned}
$$


Thus in order to find R - efficient solutions of a fuzzy $p$ - objective FMFP, we in turn obtain efficient solutions of the $3 p$ - objective MFCP, which is a basic multi objective fixed charge problem. The theorem below gives the correspondence between the set of Pareto optimal solutions of MFCP and R - efficient solutions of FMFP.

Theorem 4. A solution $X^{*} \in S$ is an $R$ - efficient solution of FMFP if and only if $X^{*}$ is an efficient solution of MFCP.

Proof. Let $X^{*} \in S$ be an R - efficient solution of FMFP. Let if possible $X^{*}$ is not an efficient solution of MFCP. Then there exists another solution $\hat{X} \in S$ such that

$$
\begin{aligned}
& Z_{k l}(\hat{X}) \leq Z_{k l}\left(X^{*}\right) \text { for all }(k, l) \in K \times\{1,2,3\} \\
& \text { and } Z_{r s}(\hat{X})<Z_{r s}\left(X^{*}\right) \text { for at least one }(r, s)
\end{aligned}
$$

Without loss of generality, we assume that the first equation is an equality constraint for all $(k, l)$ and that the second equation of strict inequality holds for exactly one index $r=k^{\prime}$ and $s=3$, say. So we have in particular,

$$
\begin{aligned}
Z_{k^{\prime} 1}(\hat{X}) & =Z_{k^{\prime} 1}\left(X^{*}\right), Z_{k^{\prime} 2}(\hat{X})=Z_{k^{\prime} 2}\left(X^{*}\right), \\
& \text { and } Z_{k^{\prime} 3}(\hat{X})<Z_{k^{\prime} 3}\left(X^{*}\right)
\end{aligned}
$$

Thus,

$$
\begin{aligned}
Z_{k^{\prime} 1}(\hat{X})+Z_{k^{\prime} 2}(\hat{X})+Z_{k^{\prime} 3}(\hat{X})<Z_{k^{\prime} 1}\left(X^{*}\right)+Z_{k^{\prime},}\left(X^{*}\right)+Z_{k^{\prime} 3}\left(X^{*}\right) \\
\text { i.e. } R\left(\tilde{Z}_{k^{\prime}}(\hat{X})\right)<R\left(\tilde{Z}_{k^{\prime}}\left(X^{*}\right)\right) \text { for } k^{\prime} \in K \\
\text { i.e. } \tilde{Z}_{k^{\prime}}(\hat{X}) \prec \tilde{Z}_{k^{\prime}}\left(X^{*}\right) \text { for } k^{\prime} \in K
\end{aligned}
$$

which contradicts that $X^{*}$ is an $\mathrm{R}$ - efficient solution of FMFP.

Conversely, let $X^{*}$ be an efficient solution of MFCP. Let if possible $X^{*}$ is not an R - efficient solution of FMFP. Then there exists another solution $\hat{X} \in S$ such that

$$
\begin{gathered}
R\left(\tilde{Z}_{k}(\hat{X})\right) \leq R\left(\tilde{Z}_{k}\left(X^{*}\right)\right) \text { for all } k \in K \\
\text { and } R\left(\tilde{Z}_{r}(\hat{X})\right)<R\left(\tilde{Z}_{r}\left(X^{*}\right)\right) \text { for at least one } r \in\{1,2, \ldots, p\}
\end{gathered}
$$

Let the strict inequality hold for some $r$. This gives

$$
1 / 3\left(Z_{r 1}(\hat{X})+Z_{r 2}(\hat{X})+Z_{r 3}(\hat{X})\right)<1 / 3\left(Z_{r 1}\left(X^{*}\right)+Z_{r 2}\left(X^{*}\right)+Z_{r 3}\left(X^{*}\right)\right)
$$

Since each component is assumed to be non negative; for at least one of $l=$ $1,2,3$ the following must hold

$$
Z_{r l}(\hat{X})<Z_{r l}\left(X^{*}\right)
$$


This shows that $X^{*}$ is not an efficient solution of MFCP, which is a contradiction. Hence proved.

If we solve each objective function of MFCP in isolation and obtain the same optimal solution for all the objectives, then an ideal solution of this problem has been attained. This rarely happens due to the conflicting nature of the objective functions. Now for real world problems, the complete solution of a multi objective problem is not always necessary to obtain and we need only a procedure which finds a compromise solution [15], [12]. A compromise solution is a solution which is preferred by the decision maker to all other solutions, taking into consideration all criteria contained in the multi objective functions. Hence, a compromise solution has to be an efficient solution. In the following section, we shall restrict our consideration to the determination of a compromise solution of MFCP on the basis of the following corollary.

Corollary 5. A compromise solution of FMFP is also a compromise solution of MFCP, and conversely.

Proof. Since the set of Pareto optimal solutions of both problems FMFP and MFCP are the same; and a compromise solution is one such Pareto optimal solution, thus the result holds.

There are three major approaches that have been developed to determine a compromise solution; namely goal programming, utility approaches and interactive procedures. The fuzzy programming approach provided by Zimmermann [14] is used here to obtain a compromise solution of the MFCP. Thus, using the min-operator and linear membership functions the resulting optimal solution of $\mathrm{LP}^{\prime}$ in Section 3.2 will always be a compromise solution of MFCP [9].

\subsection{Fuzzy Programming Approach}

In 1970, Bellman and Zadeh [3] introduced the concepts of fuzzy goal (G), fuzzy constraints (C) and fuzzy decision (D). Based on these concepts, the fuzzy decision defined by them is as $\mathrm{D}=\mathrm{G} \cap \mathrm{C}$; and this problem is characterised by the membership function $\mu_{D}=\min \left(\mu_{G}(X), \mu_{C}(X)\right)$.

In order to define the membership function of each of the multiple objectives, we compute $L_{k l}$ and $U_{k l}$ to be the lower and the upper bounds of the objective function $Z_{k l}(l=1,2,3)$ respectively. To compute these bounds, the multi objective problem MFCP is solved as a single objective fixed charge problem using each time only one objective to obtain a set of optimal solutions. For 
each objective function $Z_{k l}$, the lower bound (minimum value of $Z_{k l}$ ) $L_{k l}$ and the upper bound (maximum value of $Z_{k l}$ ) $U_{k l}$ corresponding to this set of optimal solutions is then derived. Thus, the linear membership function $\mu_{k l}\left(Z_{k l}\right)$ of the objective function is defined with respect to the upper and lower bounds as

$$
\mu_{k l}\left(Z_{k l}\right)=\left\{\begin{array}{cl}
1, & \text { if } Z_{k l} \leq L_{k l} \\
\frac{U_{k l}-Z_{k l} l}{U_{k l}-L_{k l},} & \text { if } L_{k l}<Z_{k l}<U_{k l} \\
0, & \text { if } Z_{k l} \geq U_{k l}
\end{array}\right.
$$

where $U_{k l} \neq L_{k l}, k=1,2, \ldots, p, l=1,2,3$.

In case $U_{k l}=L_{k l}$, we define $\mu_{k l}\left(Z_{k l}\right)=1$ for any value of $k, l$. Aggregating all the fuzzy sets using the minimum operator of the fuzzy decision making concept of Zimmermann [14], the corresponding single objective LP model is

(LP)

$$
\max _{X \in S} \min _{k, l} \mu_{k l}\left(Z_{k l}\right)
$$

On introducing the auxiliary variable $\beta(0 \leq \beta \leq 1)$ which represents the degree of satisfaction of the decision maker, the following linear programming problem is formulated

$\left(\mathbf{L P} \mathbf{P}^{\prime}\right.$

$$
\begin{gathered}
\max _{X \in S} \beta \\
\text { subject to } \mu_{k l}\left(Z_{k l}\right) \geq \beta, \quad \text { for all } k, l
\end{gathered}
$$

We can reduce the constraint (9) further to

$$
Z_{k l}+\beta\left(U_{k l}-L_{k l}\right) \leq U_{k l}, \quad \text { for all } k, l
$$

\subsection{Solving the Fixed Charge Problem}

Many problems of economics and logistics involve the planning of a large number of independent activities in as economical a way as is possible. To model such problems, there exists an important class of nonlinear programming problems of the form

\section{(P)}

$$
\begin{aligned}
& \operatorname{Min} Z=\sum_{j=1}^{n} c_{j} x_{j}+f_{j} y_{j} \\
& \text { subject to } \quad \sum_{j=1}^{n} a_{i j} x_{j}=b_{i}, \quad i \in I \\
& x_{j} \geq 0, \quad j \in J
\end{aligned}
$$


where $c_{j}, f_{j}(j=1,2, \ldots, n)$ are constants, and

$$
y_{j}= \begin{cases}0 & , \text { if } x_{j}=0 \\ 1 & , \text { if } x_{j}>0\end{cases}
$$

This problem with a nonlinear objective function and linear constraints is called the Fixed Charge Problem (FCP) and the constants $f_{j}$ are the fixed charges associated with the $j^{\text {th }}$ activity. These fixed charges are incurred if a new activity is engaged in at a positive level, leading to a new charge being borne by the firm.

We know that FCP is NP-hard, so we obtain an approximate solution of this NP-hard problem by using Balinski's approximation technique for FCTP [2] (which yields close to optimal solutions) and by extracting certain fixed costs [1]. Balinski showed that on relaxing the integral constraints on $y_{i j}$ and using the property

$$
y_{i j}=x_{i j} / m_{i j}, \text { where } m_{i j}=\min \left(a_{i}, b_{j}\right),
$$

and on the condition that $x_{i j} \leq m_{i j} y_{i j}$, an optimal solution exists for this relaxed problem for all pairs $(i, j)$.

In order to obtain the bounds $m_{i j}$ in the case of a FCP when the constants $a_{i}$ and $b_{j}$ are not present, we solve the following related sub-linear programs

$$
\left(\operatorname{Max} \mathbf{L P}_{j}\right) \quad \operatorname{Max}_{X \in S} z=x_{j}
$$

$$
\left(\operatorname{Min} \mathbf{L P} \mathbf{P}_{j}\right) \quad \operatorname{Min}_{X \in S} z=x_{j}
$$

Let $x_{j}^{\text {max }}$ and $x_{j}^{\text {min }}$ denote the optimal solutioms of $\operatorname{Max} L P_{j}$ and Min $L P_{j}$ respectively. Then we may say that the variable $x_{j}$ in $\mathrm{P}$ is bounded as $x_{j}^{\text {min }} \leq$ $x_{j} \leq x_{j}^{\max } \quad \forall j$.

The upper bound $x_{j}^{\max }$ is used as $m_{j}$ in the Balinski formulation, and the significance of the lower bound is that if $x_{j}^{\text {min }}>0$, then clearly the fixed charge $f_{j}$ is present in every solution and can thus be extracted from the objective function as follows [1]. Let $l$ (out of $m$ ) basic variables have have lower bound $x_{j}^{\text {min }}$ such that $x_{j}^{\text {min }}>0$. Then $\mathrm{P}$ can be rewritten as

$$
\operatorname{Min}_{X \in S} \mathrm{Z}=k \Delta+\sum_{j=1}^{n} c_{j} x_{j}+f_{j}^{*} y_{j}
$$

where $\Delta=$ coefficient of the smallest fixed charge $f_{j}, f_{j}^{*}=f_{j}-\Delta$, and $k$ is the number of non-zero basic variables, i.e. $k=m-l$. 
This extraction results in eliminating at least one variable $y_{j}$ from problem $\mathrm{P}$ and simplifies the NP-hard problem to a certain extent. A variation of Balinski's approximation can then be used on this reduced FCP to obtain tighter bounds on the objective functions as shown below. Now relax the constraints of this reduced problem by using the bounds of $x_{j}$ as $0 \leq x_{j} \leq m_{j} y_{j}$ and $0 \leq y_{j} \leq 1$, $y_{j}$ integer, $j \in J$.

Since Balinski showed that $\left\{x_{j}, y_{j}\right\}$ is a solution of this problem (ignoring integrality) if and only if $x_{j}=m_{j} y_{j}$, we formulate the problem $\mathrm{P}^{\prime}$ wherein we use this transformation and define $C_{j}=c_{j}+f_{j} / m_{j}$.

$$
\begin{aligned}
& \left(\mathbf{P}^{\prime}\right) \quad \operatorname{Min} Z^{\prime}=\sum_{j=1}^{n} C_{j} x_{j} \\
& \text { subject to } \quad \sum_{j=1}^{n} a_{i j} x_{j}=b_{i}, \quad i \in I \\
& 0 \leq x_{j} \leq m_{j}, \quad j \in J
\end{aligned}
$$

The optimal solution $\left\{x_{j}^{\prime}\right\}$ of $\mathrm{P}^{\prime}$ obtained creates bounds on the optimal value $Z^{*}$ of $\mathrm{P}$ as stated by the theorem below.

Theorem 6. [1] The optimal value $Z\left(P^{\prime}\right)$ of $P^{\prime}$ provides a lower bound to the optimal value $Z^{*}(P)$ of the corresponding FCP $P$.

In other words,

$$
\begin{gathered}
Z\left(P^{\prime}\right) \leq Z^{*}(P) \leq \sum_{j=1}^{n} c_{j} x_{j}^{\prime}+f_{j} y_{j}^{\prime} \\
\sum_{j=1}^{n} C_{j} x_{j}^{\prime} \leq Z^{*}(P) \leq \sum_{j=1}^{n} c_{j} x_{j}^{\prime}+f_{j} y_{j}^{\prime}
\end{gathered}
$$

where $y_{j}^{\prime}$ is obtained corresponding to the solution $x_{j}^{\prime}$ as

$$
y_{j}^{\prime}= \begin{cases}0 & , \text { if } x_{j}^{\prime}=0 \\ 1 & , \text { if } x_{j}^{\prime}>0\end{cases}
$$

Note Only in the case when the lower bound is equal to the upper bound in (14), then $\left\{x_{j}^{\prime}, y_{j}^{\prime}\right\}$ is an optimal solution of problem $\mathrm{P}$ with optimal value $Z^{*}(P)$. 


\section{Solution Procedure}

Step 1 Consider the given multi objective fuzzy fixed charge problem FMFP. Solve the sub-linear programs Max $L P_{j}$ and Min $L P_{j}$ to obtain bounds on the decision variables $x_{j}(j \in J)$. Relax the consraints of FMFP by introducing these upper bounds to obtain $\mathrm{FMFP}^{\prime}$.

Step 2 Corresponding to each fuzzy objective $\tilde{Z}_{k}$, introduce three crisp objective functions; $Z_{k 1}, Z_{k 2}$ and $Z_{k 3}$ by using the property of triangular distribution of fuzzy numbers. This results in a multi objective fixed charge problem which is then solved by the fuzzy programming technique for which we first obtain $U_{k l}$ and $L_{k l}$.

Step 3 To obtain these bounds on each objective function $Z_{k l}$ ( $k=$ $1, \ldots, p, l=1,2,3)$, solve the single objective fixed charge problems taking one objective at a time subject to the constraints

$$
\begin{gathered}
\sum_{j=1}^{n} a_{i j} x_{j}=b_{i}, \quad i \in I \\
0 \leq x_{j} \leq m_{j} y_{j}, \quad j \in J \\
0 \leq y_{j} \leq 1, \quad y_{j} \text { integer }, \quad j \in J
\end{gathered}
$$

where we set $m_{j}=x_{j}^{\max }$ for all $j$.

Step 4 Extract all fixed costs corresponding to those basic variables which have lower bound $x_{j}^{\text {min }}$ such that $x_{j}^{\text {min }}>0$, and set the corresponding $y_{j}=1$. Identify the smallest fixed charge say $\Delta$, corresponding to the remaining variables, and obtain (11).

Step 5 Apply Balinski transformation $x_{j}=m_{j} y_{j}$ and solve the LPP of the form $\mathrm{P}^{\prime}$ to obtain optimal solution $\left\{x_{j}^{\prime}, y_{j}^{\prime}\right\}$. Compute the values of all the objective functions at all the optimal solutions to obtain the bounds $U_{k l}$ and $L_{k l}$ on each objective $Z_{k l}$.

Step 6 Compute the membership functions $\mu_{k l}\left(Z_{k l}(X)\right)$ and introduce an auxiliary variable $\beta(0 \leq \beta \leq 1)$ to solve the equivalent mixed integer linear program LP' using LINGO. The vector $\left(\beta^{*}, X^{*}\right)$ obtained as its optimal solution gives $X^{*}$ as a compromise solution to the multi objective problem MFCP and is thus a compromise solution of FMFP.

\section{Numerical Example}

Consider the multi objective fuzzy fixed charge problem FMFP 
(FMFP) $\quad \operatorname{Min} \tilde{Z}=\left(\tilde{Z}_{1}, \tilde{Z}_{2}\right)$

$$
\begin{gathered}
\text { subject to } \\
8 x_{1}+5 x_{2}+6 x_{3}+2 x_{4}=60 \\
3 x_{1}+4 x_{2}+3 x_{3}+x_{4}=35 \\
2 x_{1}+3 x_{2}+x_{3}+2 x_{4}=20 \\
x_{1}, x_{2}, x_{3}, x_{4} \geq 0
\end{gathered}
$$

where $\tilde{Z}_{k}=\sum_{j=1}^{n}\left(\tilde{c}_{k j} x_{j}+\tilde{f}_{k j} y_{j}\right), \quad k \in K=\{1,2\}$

$$
\text { and } y_{j}= \begin{cases}0 & , \text { if } x_{j}=0 \\ 1 & , \text { if } x_{j}>0\end{cases}
$$

The triangular fuzzy costs $\tilde{c}_{k j}$ and the triangular fuzzy fixed charges $\tilde{f}_{k j}$ are given below.

$$
\begin{gathered}
\tilde{c}_{k j}=\left[\begin{array}{llll}
(7,8,9) & (1,5,11) & (1,3,5) & (2,4,6) \\
(3,6,7) & (4,8,15) & (1,4,9) & (3,7,10)
\end{array}\right] \\
\tilde{f}_{k j}=\left[\begin{array}{llll}
(20,22,27) & (24,25,26) & (21,23,25) & (20,22,28) \\
(19,21,28) & (20,27,29) & (25,26,27) & (23,25,26)
\end{array}\right]
\end{gathered}
$$

The sub-linear programs Max $L P_{j}$ and Min $L P_{j}$ are solved to obtain bounds on the decision variables $x_{j}, j \in J=\{1,2,3,4\}$,

$$
0 \leq x_{1} \leq 1.32, \quad 3.33 \leq x_{2} \leq 4.21, \quad 4.74 \leq x_{3} \leq 6.67, \quad 0 \leq x_{4} \leq 1.67
$$

On relaxing the original problem FMFP by the introduction of the upper bounds $m_{j}$ on the variables $x_{j}$, we get the problem $\mathrm{FMFP}^{\prime}$

$\left(\mathbf{F M F P}^{\prime}\right) \quad$ Minimize $\tilde{Z}=\left(\tilde{Z}_{1}, \tilde{Z}_{2}\right)$

$$
\begin{gathered}
\text { subject to } 8 x_{1}+5 x_{2}+6 x_{3}+2 x_{4}=60 \\
3 x_{1}+4 x_{2}+3 x_{3}+x_{4}=35 \\
2 x_{1}+3 x_{2}+x_{3}+2 x_{4}=20 \\
0 \leq x_{1} \leq 1.32 y_{1} \\
0 \leq x_{2} \leq 4.21 y_{2} \\
0 \leq x_{3} \leq 6.67 y_{3} \\
0 \leq x_{4} \leq 1.67 y_{4} \\
0 \leq y_{j} \leq 1, y_{j} \text { integer, } j \in J
\end{gathered}
$$


Now each fuzzy objective function $\tilde{Z}_{k}(k \in K)$ is transformed into three equivalent crisp objective functions to obtain a multi objective fixed charge problem.

$$
\begin{aligned}
& \operatorname{Min} Z_{11}=Z_{1}^{o}=7 x_{1}+x_{2}+x_{3}+2 x_{4}+20 y_{1}+24 y_{2}+21 y_{3}+20 y_{4} \\
& \operatorname{Min} Z_{12}=Z_{1}^{m}=8 x_{1}+5 x_{2}+3 x_{3}+4 x_{4}+22 y_{1}+25 y_{2}+23 y_{3}+22 y_{4} \\
& \operatorname{Min} Z_{13}=Z_{1}^{p}=9 x_{1}+11 x_{2}+5 x_{3}+6 x_{4}+27 y_{1}+26 y_{2}+25 y_{3}+28 y_{4} \\
& \operatorname{Min} Z_{21}=Z_{2}^{o}=3 x_{1}+4 x_{2}+x_{3}+3 x_{4}+19 y_{1}+20 y_{2}+25 y_{3}+23 y_{4} \\
& \operatorname{Min} Z_{22}=Z_{2}^{m}=6 x_{1}+8 x_{2}+4 x_{3}+7 x_{4}+21 y_{1}+27 y_{2}+26 y_{3}+25 y_{4} \\
& \operatorname{Min} Z_{23}=Z_{2}^{p}=7 x_{1}+15 x_{2}+9 x_{3}+10 x_{4}+28 y_{1}+29 y_{2}+27 y_{3}+26 y_{4}
\end{aligned}
$$

$$
\text { subject to }(14)-(21)
$$

To solve this multi objective program, we use the fuzzy programming approach and first obtain the bounds $U_{k l}$ and $L_{k l}$ on each objective $Z_{k l}$ by solving one objective function at a time. Consider

$$
\operatorname{Min} Z_{13}=9 x_{1}+11 x_{2}+5 x_{3}+6 x_{4}+27 y_{1}+26 y_{2}+25 y_{3}+28 y_{4}
$$

$$
\text { subject to }(14)-(21)
$$

Extracting the fixed charges for the variables $x_{2}^{\text {min }}, x_{3}^{\text {min }}>0$ gives $y_{2}=$ $y_{3}=1$, and the objective function is revised as

$$
\operatorname{Min} Z_{13}=26+25+9 x_{1}+11 x_{2}+5 x_{3}+6 x_{4}+27 y_{1}+28 y_{4}
$$

Choose $\Delta=27$ (the smallest remaining $f_{j}$ ). Here the number of basic variables $m$, is 3 . Since two basic variables have already been identified with $x_{j}>0$, only $k=(m-l)=1$ more basic variable needs to be determined. Thus the resulting objective function is

$$
\begin{aligned}
\operatorname{Min} Z_{13} & =51+27+9 x_{1}+11 x_{2}+5 x_{3}+6 x_{4}+(27-27) y_{1}+(28-27) y_{4} \\
& =78+9 x_{1}+11 x_{2}+5 x_{3}+6 x_{4}+y_{4}
\end{aligned}
$$

Using Balinski transformation $y_{4}=x_{4} / m_{4}$, the modified objective function becomes

$$
\operatorname{Min} Z_{13}=78+9 x_{1}+11 x_{2}+5 x_{3}+(6+1 / 1.67) x_{4}
$$

and the corresponding LP problem $\mathrm{P}^{\prime}$ becomes 
$\left(\mathbf{P}^{\prime}\right) \quad$ Minimize $Z_{13}^{\prime}=78+9 x_{1}+11 x_{2}+5 x_{3}+6.6 x_{4}$

subject to $\quad 8 x_{1}+5 x_{2}+6 x_{3}+2 x_{4}=60$

$$
\begin{gathered}
3 x_{1}+4 x_{2}+3 x_{3}+x_{4}=35 \\
2 x_{1}+3 x_{2}+x_{3}+2 x_{4}=20 \\
x_{1}, x_{2}, x_{3}, x_{4} \geq 0
\end{gathered}
$$

We obtain the solution $X_{3}^{\prime}=(0,3.33,6.67,1.67)$ with $Z_{13}^{\prime}\left(X_{3}^{\prime}\right)=159$. Set $y_{2}^{\prime}=y_{3}^{\prime}=y_{4}^{\prime}=1$ and $y_{1}^{\prime}=0$, then the value of the corresponding objective function of the FCP is $Z_{13}\left(X_{3}^{\prime}\right)=159$. Since the optimal values of the relaxed integer function $\mathrm{P}^{\prime}$ and the regular fixed charge function $\mathrm{P}$ are the same, we get that $X_{3}^{\prime}$ is a global minimum.

On solving the remaining objective functions similarily, we obtain the following optimal solutions

$$
\begin{aligned}
& X_{1}^{\prime}=(0,3.33,6.67,1.67) ; Z_{11}^{\prime}\left(X_{1}^{\prime}\right)=78.34, Z_{11}\left(X_{1}^{\prime}\right)=78.34 \\
& X_{2}^{\prime}=(0,3.33,6.67,1.67) ; Z_{12}^{\prime}\left(X_{2}^{\prime}\right)=113.34, Z_{12}\left(X_{2}^{\prime}\right)=113.34 \\
& X_{4}^{\prime}=(1.32,4.21,4.74,0) ; Z_{21}^{\prime}\left(X_{4}^{\prime}\right)=89.53, Z_{21}\left(X_{4}^{\prime}\right)=89.53 \\
& X_{5}^{\prime}=(1.32,4.21,4.74,0) ; Z_{22}^{\prime}\left(X_{5}^{\prime}\right)=134.53, Z_{22}\left(X_{5}^{\prime}\right)=134.56 \\
& X_{6}^{\prime}=(1.32,4.21,4.74,0) ; Z_{23}^{\prime}\left(X_{6}^{\prime}\right)=197.53, Z_{23}\left(X_{6}^{\prime}\right)=199.05
\end{aligned}
$$

Thus, $X_{A}=(0,3.33,6.67,1.67)$ and $X_{B}=(1.32,4.21,4.74,0)$ are the only solutions obtained, and the value of every objective function computed at each of these two points will provide the bounds $U_{k l}$ and $L_{k l}$ as follows

$$
\begin{array}{ll}
L_{11}=Z_{11}\left(X_{A}\right)=78.34 & U_{11}=Z_{11}\left(X_{B}\right)=83.19 \\
L_{12}=Z_{12}\left(X_{A}\right)=113.34 & U_{12}=Z_{12}\left(X_{B}\right)=115.83 \\
L_{13}=Z_{13}\left(X_{A}\right)=159 & U_{13}=Z_{13}\left(X_{B}\right)=159.89 \\
L_{21}=Z_{21}\left(X_{B}\right)=89.53 & U_{21}=Z_{21}\left(X_{A}\right)=97 \\
L_{22}=Z_{22}\left(X_{B}\right)=134.56 & U_{22}=Z_{22}\left(X_{A}\right)=143.01 \\
L_{23}=Z_{23}\left(X_{B}\right)=199.05 & U_{23}=Z_{23}\left(X_{A}\right)=208.68
\end{array}
$$

On computing the membership functions corresponding to these six objective functions and on introducing the auxiliary variable $\beta$, we obtain the following mixed integer linear program $\mathrm{LP}^{\prime}$

$$
\begin{aligned}
& \left(\mathbf{L P}^{\prime}\right) \quad \max _{X \in S} \beta \quad \text { subject to } \\
& 4.85 \beta+7 x_{1}+x_{2}+x_{3}+2 x_{4}+20 y_{1}+24 y_{2}+21 y_{3}+20 y_{4} \leq 83.19
\end{aligned}
$$




$$
\begin{gathered}
2.49 \beta+8 x_{1}+5 x_{2}+3 x_{3}+4 x_{4}+22 y_{1}+25 y_{2}+23 y_{3}+22 y_{4} \leq 115.83 \\
0.89 \beta+9 x_{1}+11 x_{2}+5 x_{3}+6 x_{4}+27 y_{1}+26 y_{2}+25 y_{3}+28 y_{4} \leq 159.89 \\
7.47 \beta+3 x_{1}+4 x_{2}+x_{3}+3 x_{4}+19 y_{1}+20 y_{2}+25 y_{3}+23 y_{4} \leq 97 \\
8.45 \beta+6 x_{1}+8 x_{2}+4 x_{3}+7 x_{4}+21 y_{1}+27 y_{2}+26 y_{3}+25 y_{4} \leq 143.01 \\
9.63 \beta+7 x_{1}+15 x_{2}+9 x_{3}+10 x_{4}+28 y_{1}+29 y_{2}+27 y_{3}+26 y_{4} \leq 208.68
\end{gathered}
$$

Using LINGO, we get a compromise solution as $\beta^{*}=0.11, x_{1}^{*}=0, x_{2}^{*}=3.33$, $x_{3}^{*}=6.67, x_{4}^{*}=1.67, y_{1}^{*}=0, y_{2}^{*}=1, y_{3}^{*}=1, y_{4}^{*}=1$, and the corresponding fuzzy nondominated solution is $\tilde{Z}_{1}=(78.34,113.34,159), \tilde{Z}_{2}=(93,142.99,208.68)$ and the overall optimised satisfaction $\beta^{*}=0.11$.

Note The compromise solution we have obtained above is the same as $X_{A}$. On further inspection, we find that $X_{B}=(1.32,4.21,4.74,0)$ is also an $\mathrm{R}$ efficient solution of the original problem FMFP.

\section{Conclusion}

In this paper we have obtained a solution procedure for solving the multi objective fuzzy fixed charge problem. Rather than obtaining the set of all efficient solutions, we have obtained a compromise solution to this problem. The compromise solution obtained is the most preferred efficient solution to the decision maker as it maximises his level of satisfaction. Further work to be done in this paper is to explore other fuzzy aggregation operators which will best represent the decision maker's fuzzy preferences.

\section{Acknowledgements}

The Author ${ }^{1}$ is thankful to the Council of Scientific and Industrial Research (CSIR) for providing financial assistance for this paper.

\section{References}

[1] V. Adlakha, K. Kowalski, A heuristic algorithm for the fixed charge problem, Opsearch, 47, No. 2 (2010), 166-175, doi: 10.1007/s12597-010-0016-z. 
[2] M.L. Balinski, Fixed cost transportation problems, Naval Research Logistics Quarterly, 8 (1961), 4154, doi: 10.1002/nav.3800080104.

[3] R. E. Bellman, L. A. Zadeh, Decision-making in a fuzzy environment, Management Science, 17 (1970), 141164, doi: 10.1287/mnsc.17.4.B141.

[4] L. Cooper, The fixed charge problemI: A new heuristic method, Computers and Mathematics with Applications, 1 (1975), 89-95, doi: 10.1016/08981221(75)90010-3.

[5] L. Cooper, C. Drebes, An approximate solution method for the fixed charge problem, Naval Research Logistics Quarterly, 14 (1967), 101-113, doi: 10.1002/nav.3800140110.

[6] D. Dubois, H. Prade, Fuzzy Sets and Systems. Theory and Applications, Academic (1980).

[7] W.M. Hirsch, G.B. Dantzig, The fixed charge problem, Naval Research Logistics Quarterly, 15 (1968), 413-424, doi: 10.1002/nav.3800150306.

[8] H.G. Kocken, M. Ahlatcioglu, A compensatory approach to multiobjective linear transportation problem with fuzzy cost coefficients, Mathematical Problems in Engineering, 2011 (2011), 1-19, doi: 10.1155/2011/103437.

[9] H. Leberling, On finding compromise solutions in multicriteria problems using the fuzzy-min operator, Fuzzy Sets and Systems 6 (1981), 105-118, doi: 10.1016/0165-0114(81)90019-1.

[10] K.G. Murty, Solving the fixed charge problem by ranking the extreme points, Operations Research, 16 (1968), 268-279, doi: 10.1287/opre.16.2.268.

[11] D.I. Steinberg, The fixed charge problem, Naval Research Logistics Quarterly, 17 (1970), 217-235, doi: 10.1002/nav.3800170209.

[12] W.F.A. El-Wahed, A multi-objective transportation problem under fuzziness, Fuzzy Sets and Systems, 117 (2001), 27-33, doi: 10.1016/S01650114(98)00155-9.

[13] W.E. Walker, A heuristic adjacent extreme point algorithm for the fixed charge problem, Management Science, 22 (1976), 587-596, doi: $10.1287 /$ mnsc.22.5.587. 
[14] H.J. Zimmermann, Fuzzy programming and linear programming with several objective functions, Fuzzy Sets and Systems, 1 (1978), 45-55, doi: 10.1016/0165-0114(78)90031-3.

[15] H.J. Zimmermann, Applications of fuzzy set theory to mathematical programming, Information Sciences, 36 (1985), 29-58, doi: 10.1016/00200255(85)90025-8. 